\title{
Developing an Optimal Experimental Model for Immunological Studies in VCA: Vascularized Composite Allotransplants Based on Femoral Vessels in Rats
}

\author{
Andreea Grosu-Bularda, Dragos Zamfirescu, Andrei Stefanescu, Marius Stoica, Luana Lazarescu, \\ Ioan Lascar
}

Clinic of Plastic Surgery, Aesthetic and Reconstructive Microsurgery,

Emergency Clinical Hospital, Bucharest, Romania

\author{
Corresponding author: \\ Andreea Grosu-Bularda, MD \\ Clinic of Plastic Surgery, Aesthetic \\ and Reconstructive Microsurgery, \\ Emergency Clinical Hospital, Calea \\ Floreasca no.8, 014461, Bucharest \\ Romania \\ E-mail: andreeabularda@gmail.com
}

\section{ABSTRACT}

Vascularized composite allotransplantation (VCA) has become a recent therapeutic entity in reconstructive surgery. Long term immunosuppressive therapy ensures graft survival, although, with a risk of serious side effects. Further progress in this field depends on better understanding of basic immunology of composite tissue grafts with the dynamics of rejection and possibilities to induce donor specific-tolerance. Without a vast clinical experience, a possibility for achieving those goals is intensive study in preclinical field. The classical experimental model for VCA is rat hind limb transplantation. We analyzed several microsurgical models in order to determine the most adequate one for immunological studies. We performed both orthotopic and heterotopic hind limb transplantations (we tested different techniques: entire or partial hind limb allotransplantation) and also osteomyocutaneous flaps (with different bone components), noting their advantages and limits. The osteomyocutaneous flap is less morbid and represents a promising experimental model in VCA studies. Also we analyzed other models including epigastric flap allotransplantation and abdominal wall transplant for determine their utility as experimental models for immunological studies.

Key words: rat model immunological studies, hind limb allotransplantation, epigastric flap, hemi-abdominal wall, vascularized composite allotransplantation

\section{INTRODUCTION}

Vascularized Composite Allotransplantation (VCA) is an emerging new dimension in transplant medicine, offering a viable reconstructive option for injuries and defects involving multiple layers of functional tissue, impossible to repair using conventional surgical techniques (1). Since 1998, when first hand transplant was performed, vascularized composite allotransplantation became a new clinical entity, with different types of reconstructive transplants performed 
for various anatomic locations including: upper and lower extremities, face, larynx, tongue, trachea, abdominal wall, uterus, penis. Reported short- and long-term outcomes have been excellent thus far, reconstructive transplantation is now a reality, and basic and translational research in this field is advancing on many fronts, in order to overcome the immunological challenge (2-6).

The immunology of composite tissue allotransplantation is complex because the grafts are composed of a variety of tissues, such as skin, fat, muscle, nerves, lymph nodes, bone, cartilage, ligaments, and bone marrow, representing different antigenicities, with skin being the most antigenic tissue (7). Recent advances in transplant immunology are shifting the focus from immunosuppression to immunoregulation, making composite tissue allotransplantation with novel and less potent immunosuppressive regimens a possibility. The long-term goal is to induce donor-specific tolerance in order to avoid the toxicity of immunosuppressive drugs. Acceptance of the graft in absence of immunosuppressive therapy have been termed the "holy grail" in transplantation and would represent a transformational achievement for using vascularized composite allotransplantation as reconstructive option for extensive defects, allowing unlimited potential for tissue repair $(3-5,8)$.

Working on animal models permits the familiarization with microsurgical techniques in experimental composite tissue allotransplantation and evaluation of important immunological aspects: rejection dynamics evaluation, immunosuppresion protocol testing and induction of immunologic tolerance. As small animal model, the rat is a good option for VCA studies in order to furher establish a complex translational program (9).

In order to reduce the duration of the procedures, morbidity and mortality of the animals and evaluating long term results of the immunologic protocols applied, we have primarily regarded setting an adequate experimental model for immunologic studies, implying simple models of vascularized composite transplants.

\section{AIM OF THE STUDY}

Our purpose was to develop a robust animal model that allows evaluation of multiple parameters involved in the allotransplantation of vascularized composite tissue. We want to standardize our knowlledge in order to obtain the most suitable animal model to perform immunological studies, for reducing and refining the immunosupressive therapy and promoting transplant tolerance. For evaluating immunological aspects we need a simple animal model, with less surgical technical difficulties, less complications and long term rates of survival.

\section{MATERIALS AND METHODS}

\section{Presentation of the experimental activity}

Before starting the proper experimental activity, certain conditions were attained: a compelling research activity, work protocols elaboration and acquisition of laboratory animals and all disposable items.

Surgical interventions and postoperatory follow-up of the animals during the study have been performed in the Experimental Microsurgery Laboratory of the Plastic Surgery Department of the Bucharest Clinical Emergency Hospital.

The laboratory animals included in this study received adequate treatment, according to national and european ethic regulations. We were concerned that the number of tested animals and their sufferance be reduced to a minimum, and their sacrifice has been performed accordingly, not painfull and in agreement to internal conduit regulations of the laboratory, insuring adequate anesthesia, analgesia and general protection towards pain. All rats have been given the same living conditions, standardized, pre- and post-operatory, insuring thermal comfort, food and water ad libitum.

The experimental animals were male rats pertaining to two unrelated breeds: Brown Norway and Lewis, with high antigenic incompatibility - highly useful aspect for evaluating immunological parameters in transplant. We have used animals with the age of 13-14 weeks, weight: 250-350 grams, the size of the animal being important due to the vascular pedicle size, a larger vascular caliber facilitating microvascular anastomosis. The following surgical transplant methods of the hind limb were analyzed: orthotopic and heterotopic transplant and osteomyocutaneous flap transplant. Also surgical models of epigastric flap allotransplantation and abdominal wall transplantation were analyzed. The vascularized composite allotransplants were performed between Brown Norway donor rats and Lewis recipients. We have used Cyclosporin $A$ in a dose of 16 $\mathrm{mg} / \mathrm{kg}$ body weight for preventing the rejection. In couple of cases we used syngenic transplant models (between rats of the same breed), only to get used with the surgical technique, not for immunological purpose.

The table 1 presents the procedures that were performed.

It is preferable that the surgical interventions on both the donor and the recipient be performed at the 
Table 1

\begin{tabular}{|c|c|c|}
\hline Procedure & Description & Number of procedures \\
\hline Orthotopic hind limb allotransplant & Harvesting at trans-femoral level & 3 \\
\hline Heterotopic partial hind limb allotransplant & Recipient area-dorsal aspect of the hip & 7 \\
\hline Heterotopic entire hind limb allotransplant & Recipient area-inguinal region(syngenic model) & 1 \\
\hline \multirow[t]{3}{*}{ Osteomyocutaneous flap allotransplant } & Bone component: the femur & 7 \\
\hline & Knee transplant & 2 \\
\hline & Calf transplant & 2 \\
\hline Epigastric flap transplantation & Syngenic model & 4 \\
\hline Raising of hemi-abdominal wall flap & Full thickness, all the layers of abdominal wall & 4 \\
\hline
\end{tabular}

same time, by two surgeons, in order to reduce the operating time and the ischemia time (when a single surgeon performs the intervention, a rigorous preparation is needed, raising the allograft of the donor rat preparing the vascular pedicle, dissecting the sciatic nerve, disarticulation of the limb, then preparing of the recipient site in the Lewis rat, returning to the donor rat, section of the vascular pedicle of the hind limb, harvesting it and performing the transplant on the donor, following the steps mentioned lower).

\section{Orthotopic hind limb transplantation}

The surgical technique was based on the models previously described in the literature (10).

The steps of the orthotopic allotransplant of the hind limb are:

- preparing the surgery;

- anesthesia of the experimental animals;

- intervention in the donor animal:

- skin incision;

- dissection of the femoral vascular pedicle and the femoral nerve;

- section of the musculature of the thigh;

- dissection of the sciatic nerve;

- section of the femur.

Section of the vascular pedicle and detaching the hind limb of the donor rat is performed after the preparation of the recipient area of the Lewis rat.

- intervention in the recipient rat: the same steps are followed for the dissection and identification of the elements, having the following important aspects: preservation of a higher length of the vascular pedicle, with a more distal section of it (in order to avoid tension in anastomosis), more distal cutting of the sciatic nerve for avoid tension nerve suture, as well as maintaining a better part of the hip muscle mass in order to restore as much as possible the function of the transplanted limb;
- section of the vascular pedicle and harvesting the hind limb from the donor;

- proper transplant procedure includes:

- osteosynthesis of the femur: axial fixation and stabilization through cerclage;

- suture of the deep muscles of the thigh;

- direct coaptation of the sciatic nerve ends and epiperineural microsurgical nerve repair under surgical microscope;

- microvascular anastomosis, with separate sutures under surgical microscope of the femoral vessels;

- microsurgical restoration of the femoral nerve;

- completing the repair of the musculature;

- skin sutures.

The fig. 1-5 show the steps of the orthotopic transplant of the hind limb.

\section{The heterotopic transplant of the hind limb}

It was important to develop testing of new experimental models of non-functional hind limb transplant, also supported by the femoral vascular pedicle, heterotopic models-the recipient site being in a different region than the anatomical one, with the purpose of simplifying the surgical procedure and reducing the complications rate.

The hind limb of the donor rat can be harvested from different levels, and its positioning in the recipient may also differ in the case of different experimental models, therefore there are more types of heterotopic transplant described in literature (11-13).

We tested two models, in order to identify their advantages and disadvantages:

1. Harvesting the hind limb of the donor is performed at knee level, and its positioning in the recipient is in the dorsal region of the hip, in a hardly accessible area of the animal (fig. 6).

There are important technical details like: 


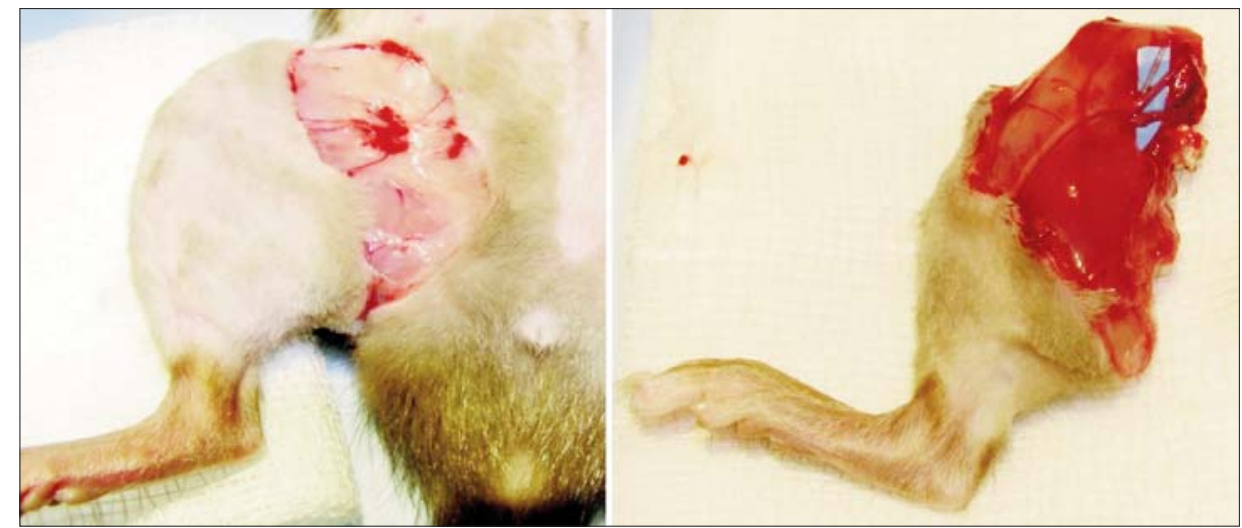

Figure 1 - Harvesting the allograft from the donor rat

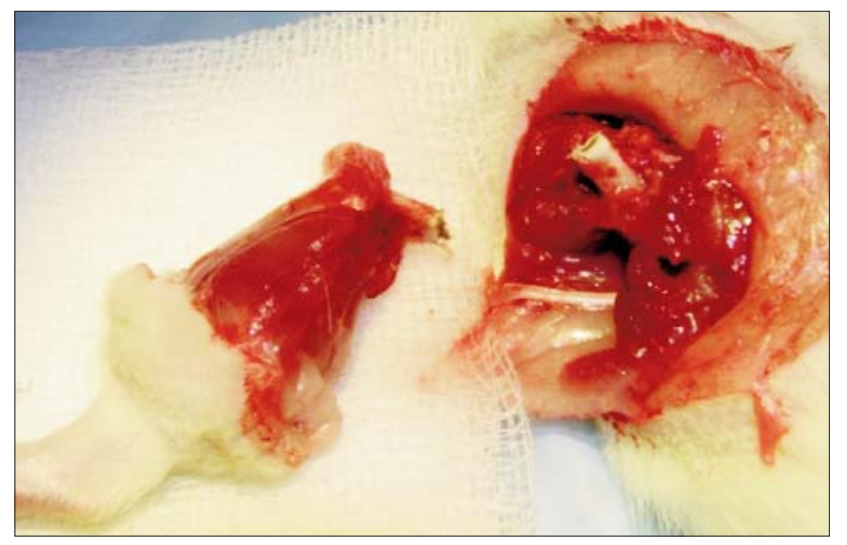

Figure 2 - Removing the hind limb from the recipient rat

- adequate, stabile fixation of the hind donor limb in the soft tissue from the dorsal region of the recipient's hip;

- creating a tunnel/groove for placing the anastomosed vascular pedicles, an area that has to be large enough in order to prevent the compression of the vessels;

- the vascular pedicles must be long enough for preventing the tension in the suture, but a correct positioning is also necessary for avoiding their kinking;

- control of the hemostasis is also an important aspect.

2. A different type of heterotopic transplant was tested( described by Ulusal and co.) and it implies harvesting the entire hind limb of the donor rat, as well as the epigastric flap in the inguinal region of the recipient (11).

We have tested this experimental model on a syngenic model with the aim of familiarizing with the surgical procedure (fig. 7-10).

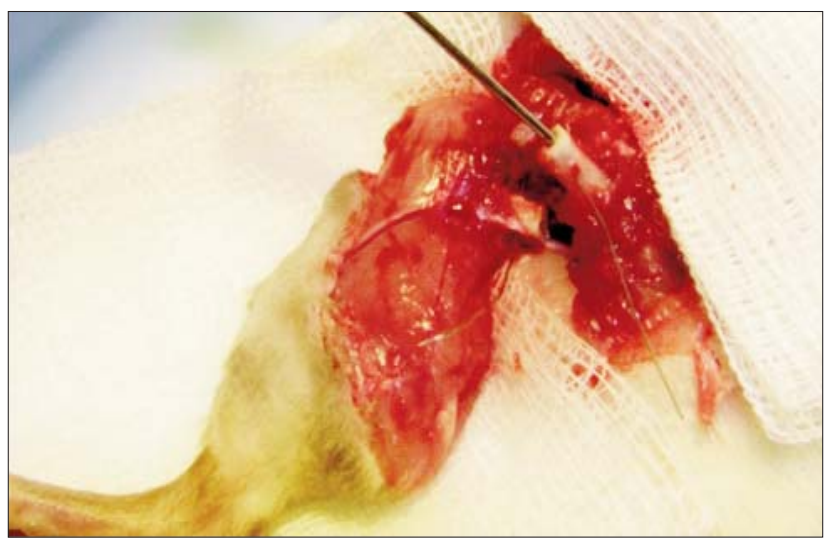

Figure 3 - Osteosynthesis of the femur

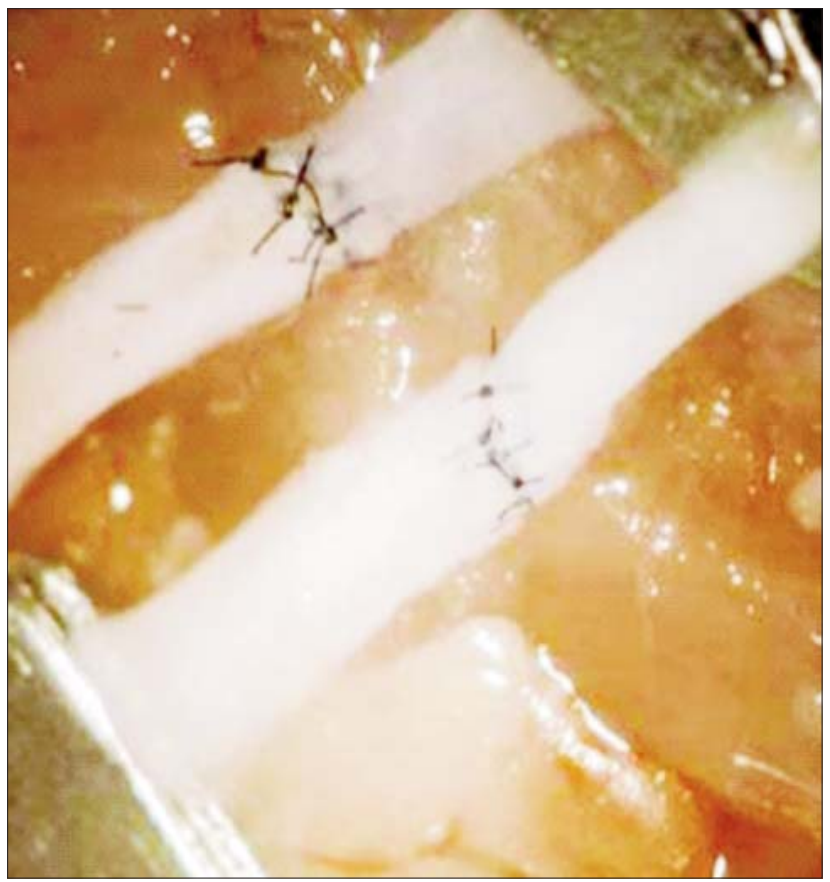

Figure 4 - Performing microvascular anastomosis 


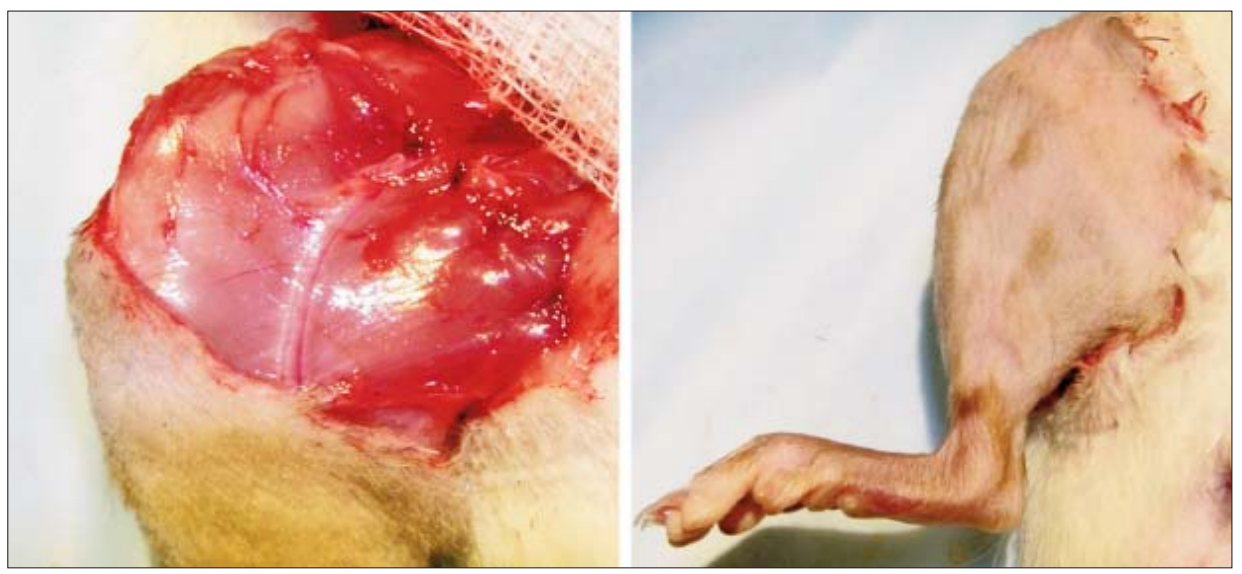

Figure 5 - Muscle repair and skin suture - final aspect of the transplant

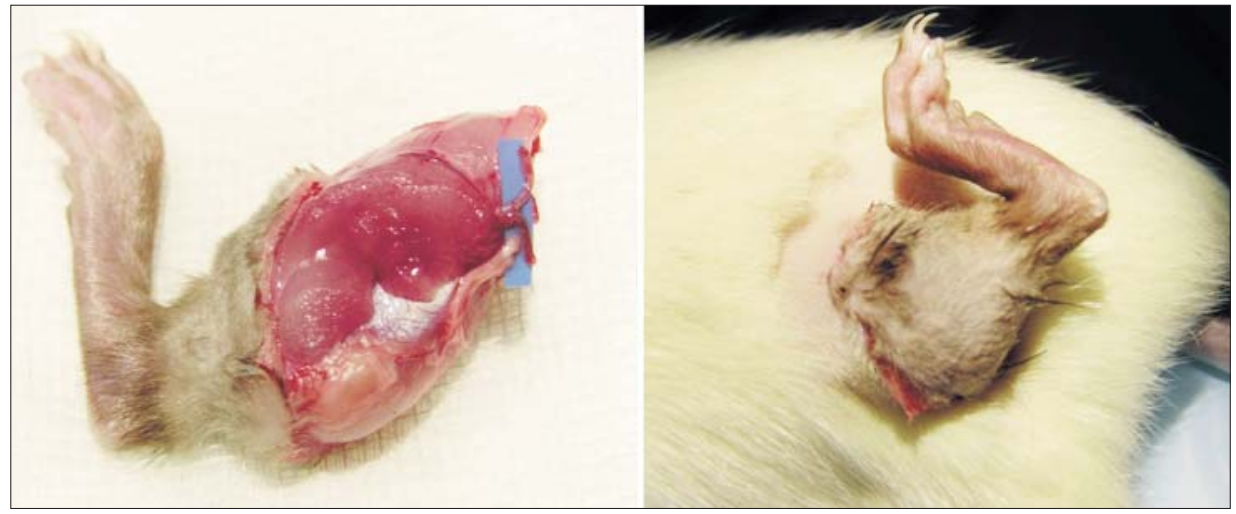

Figure 6 - Heterotopic partial hind limb allotransplantation

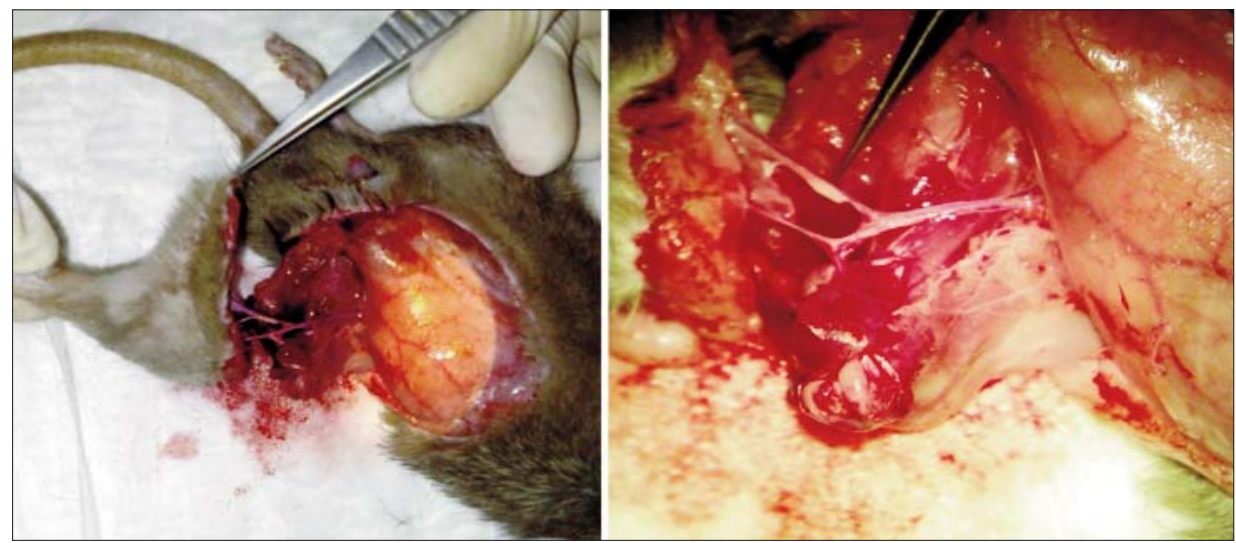

Figure 7 - Harvesting the hind limb + epigastric flap

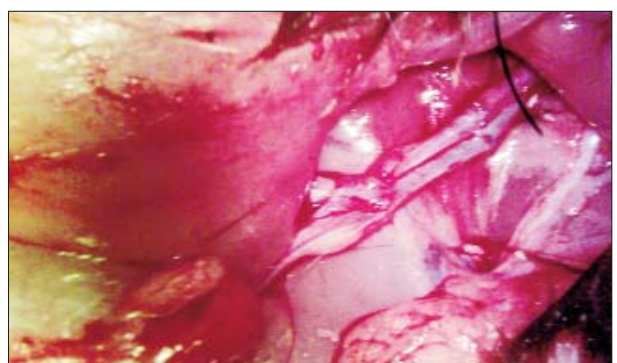

Figure 8 - Microvascular anastomosis have been performed on the femoral vessels

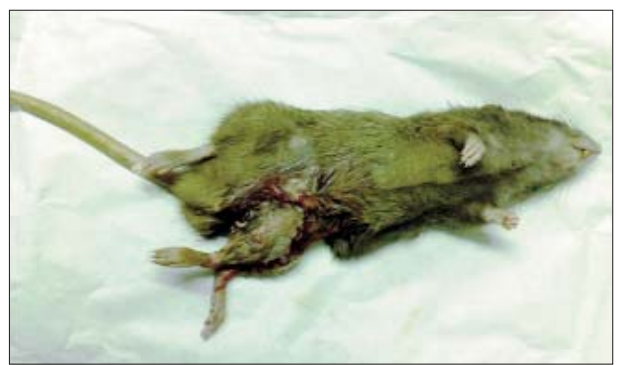

Figure 9 - The hind limb of the donor has been fixated in the inguinal region of the recipient 


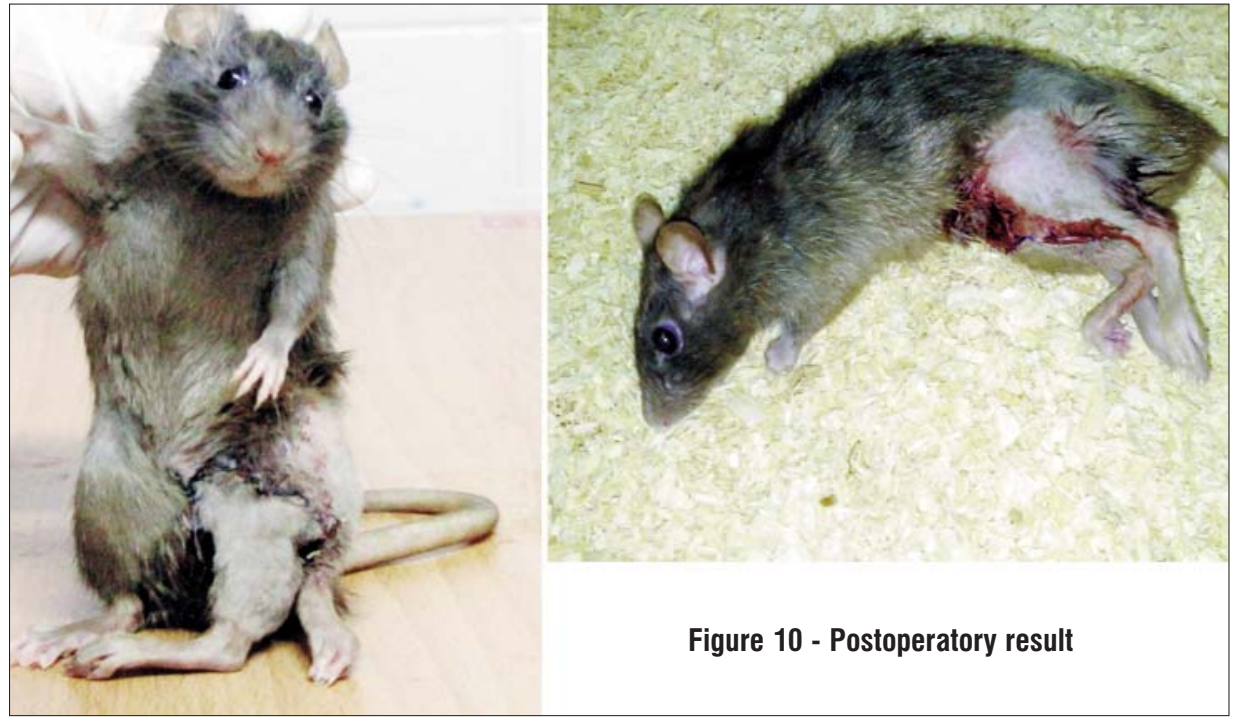

\section{Allotransplantation of osteomyocutaneous} flap, based on femoral vessels.

An alternative solution for transplantation of entire /partial hind limb is to transplant osteomyocutaneous flap, based on femoral vessels, with different bony component (13-15).
We illustrate bellow the models that we tested for osteomyocutaneous flap allotransplants.

1. One of these models is the osteomyocutaneous femoral flap, which has the whole donor's femur, the transplantation being performed in the inguinal region of the recipient This procedure is presented in fig. 11-12.

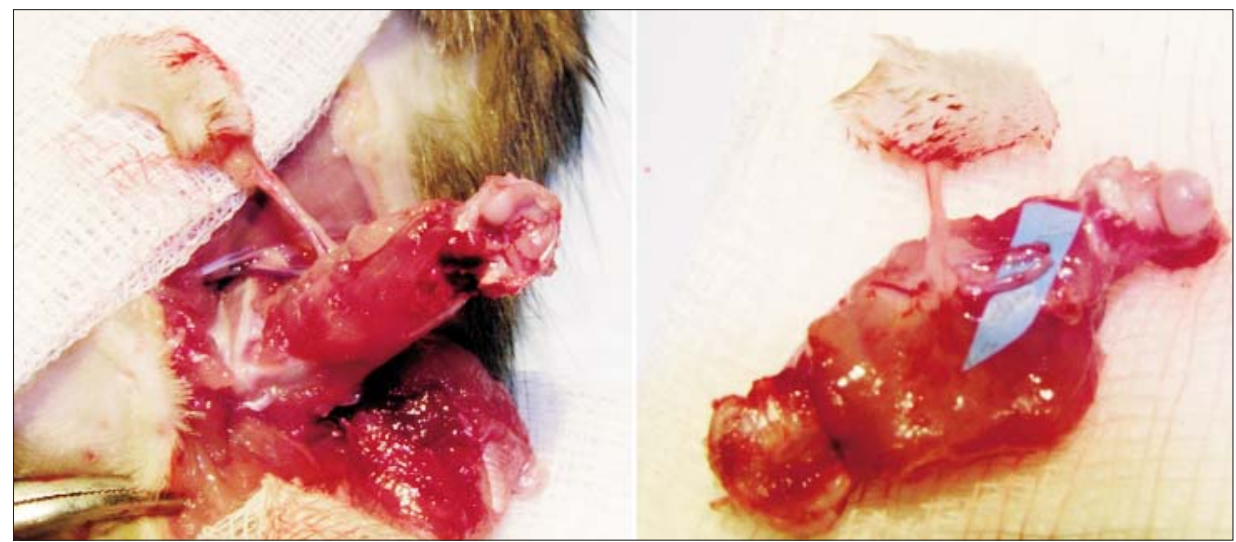

Figure 11 - 0steomyocutaneous flap being prepared for transplant

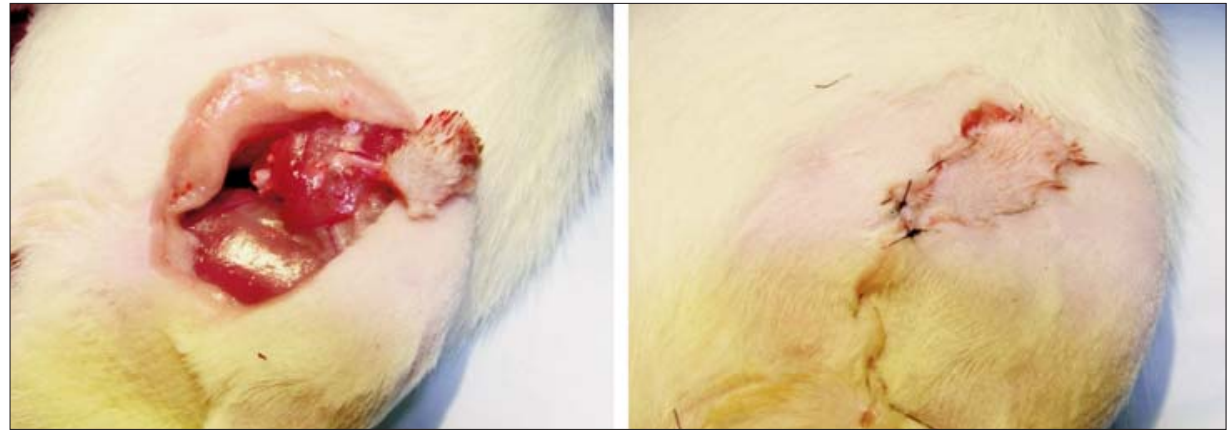

Figure 12 - Transplantation of the allograft and immediate postoperatory aspect 


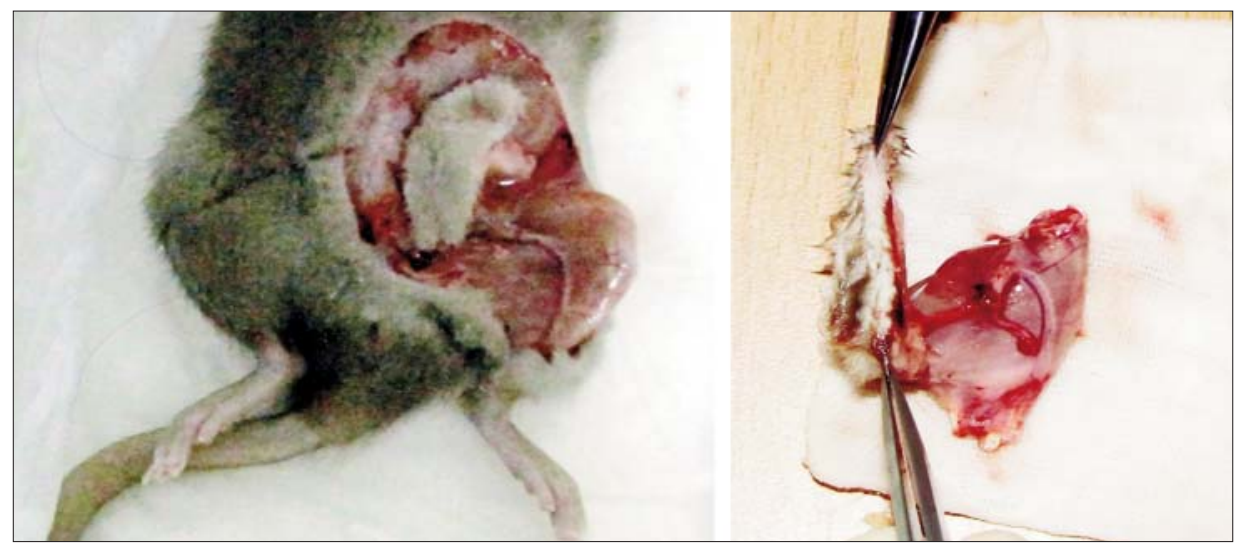

Figure 13 - Raising of the allograft

2. Another model is represented by the calf osteomiocutaneous transplantation, as we can see in fig. 13, 14.

3. Osteomyocutaneous knee allotransplants, placed as well in the inguinal region of the recipient rat simillar to the models described above, were performed (fig. 15).

In order to estimate which of the three transplant models based on femoral vessels (orthotopic, heterotopic transplantation and osteomyocutaneous flap) are most suitable for immunological studies we analyzed three experimental groups:

- Group 1: hind limb orthotopic allotransplantation $(n=3)$;

- Group 2: partial hind limb heterotopic allotransplantation $(n=7)$;

- Group 3: femoral osteomyocutaneous flap allotransplantation $(n=7)$.

All the allotransplantations were done between Brown Norway donors and Lewis recipients lab rats treated in standardized conditions. The procedures were conducted by the same surgical team following a well established protocol. Cyclosporine (CsA) $16 \mathrm{mg} / \mathrm{kg}$ was used as post-operative immunosuppression.

We watched the assessment of surgical technique objective parameters: comparing total operatory time and ischemia time for each procedure.

The graphics 1, 2 show the average surgical and ischemia times. The osteomyocutaneous flap was the most time saving procedure (average surgical time 109 minutes, followed by heterotopic and orthotopic transplantations procedures with 129 minutes and 163 minutes respectively. Ischemia time values were: 77 minutes (orthotopic transplantation), 49 minutes (heterotopic one) and 35 minutes (osteomyocutaneous flap procedure).

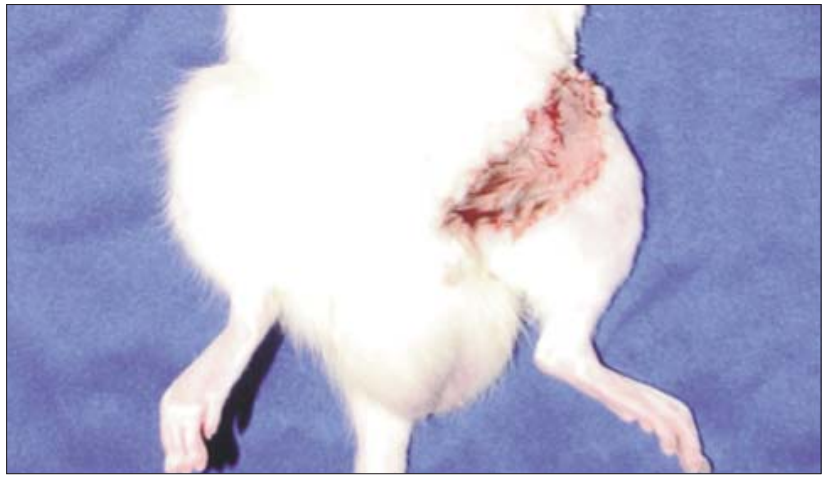

Figure 14 - Immediate post-operatory aspect

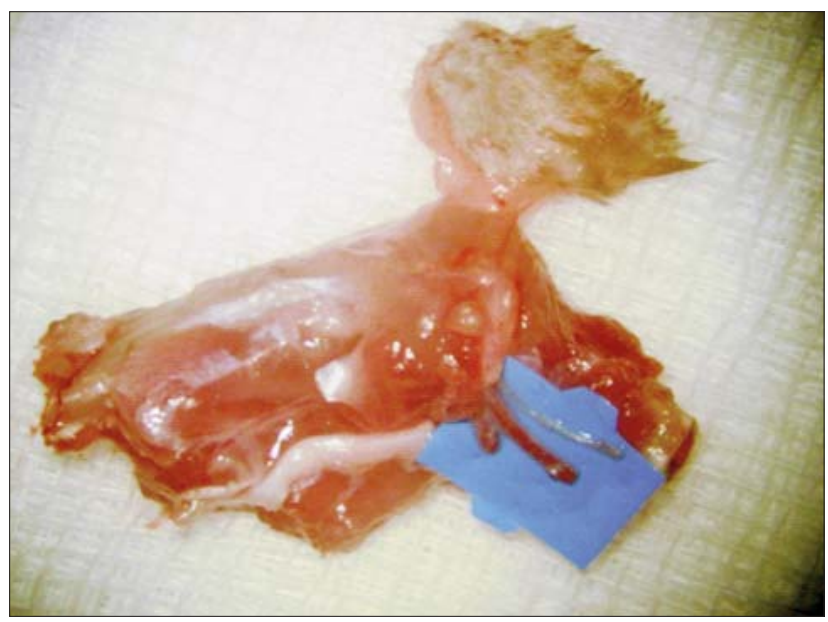

Figure 15 - Osteomyocutaneous knee allograft

Besides the hind limb transplantation experimental models shown (all having bone tissue component included) the soft tissue (no bone tissue component included) flaps based on femoral vessels were also kept in sight. The epigastric flap transplantation is an example of the latter experimental model. 


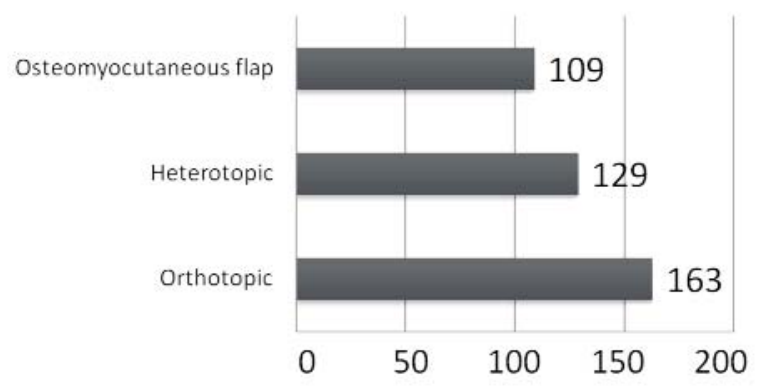

Graphic 1 - Mean operating time (min)
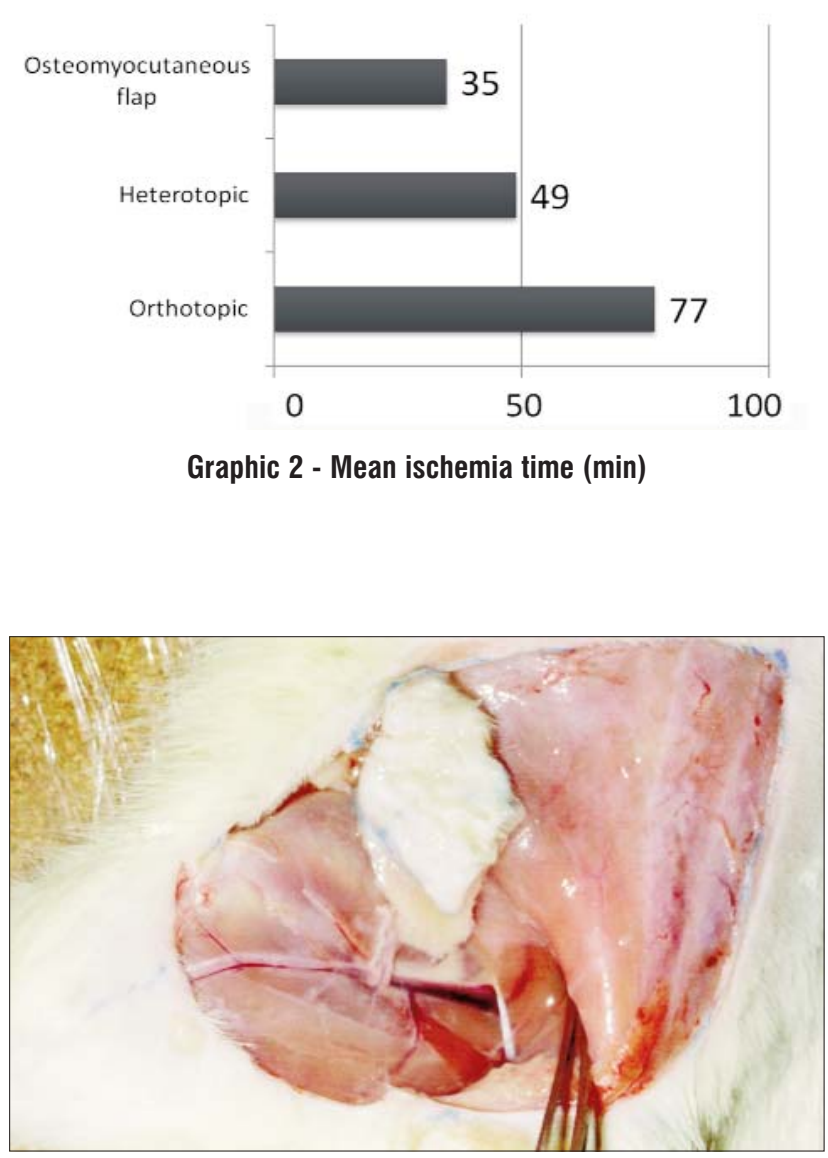

Figure 16 - Flap dissection in the donor rat

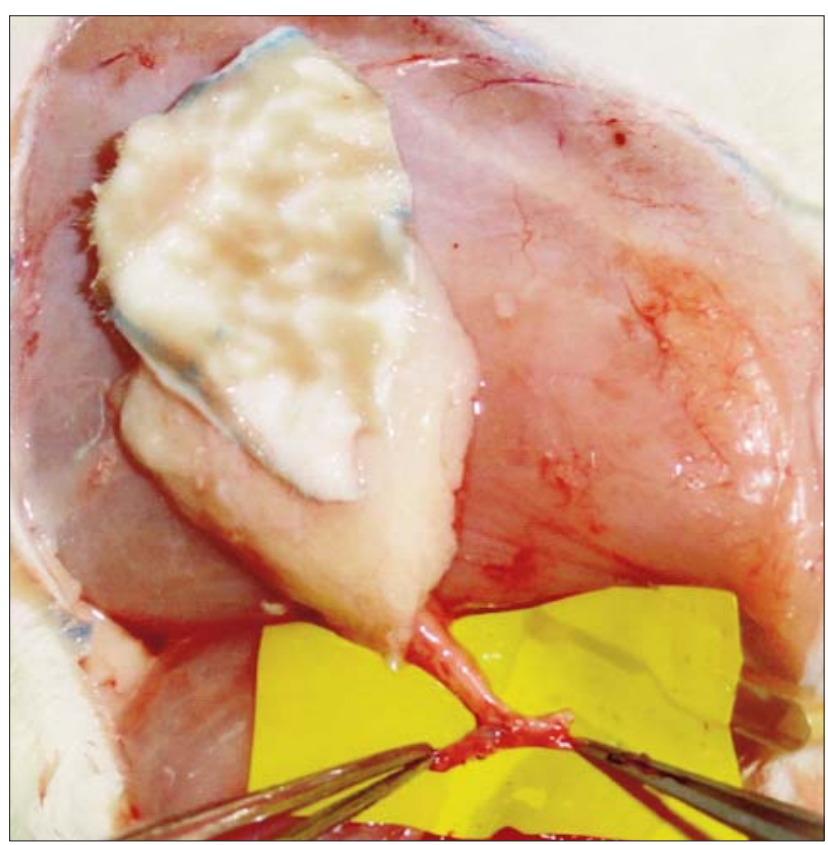

Figure 17 - Raising the flap based on femoral vessels

median line, a less vascularized region. Stiches are placed on the margins of the flap to avoid shearing of the component layers. 


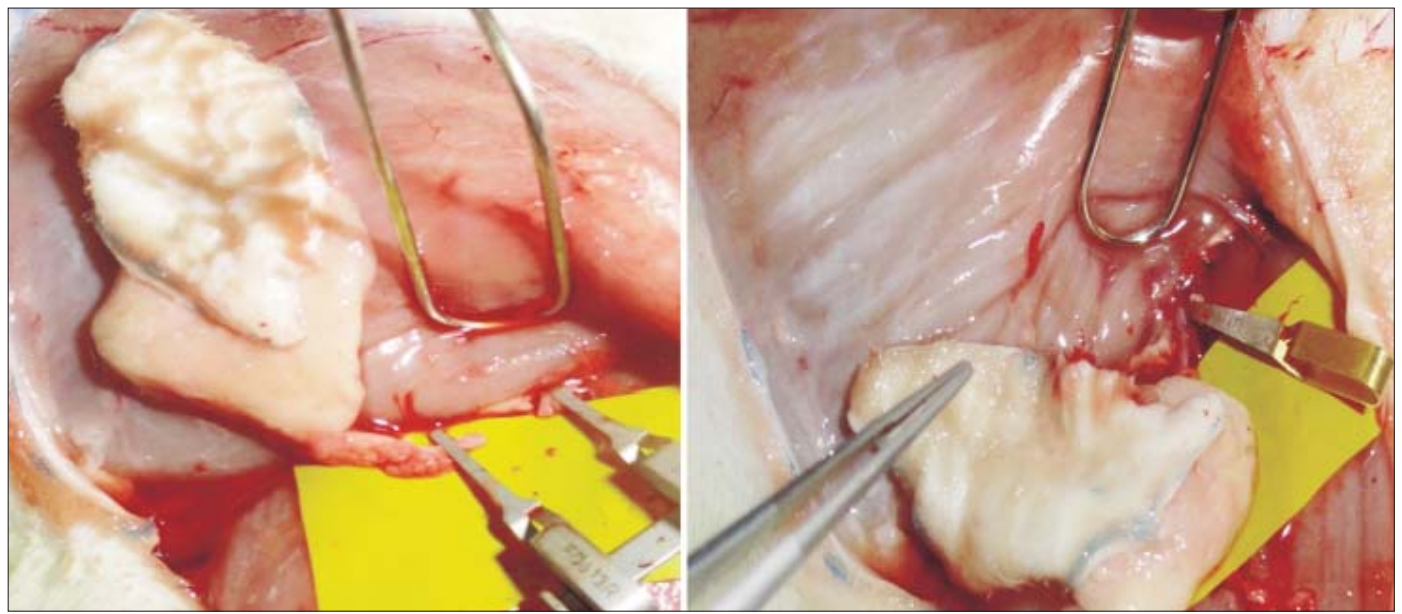

Figure 18 - Preparation for microvascular anastomoses (syngenic model) and the afterwards aspect

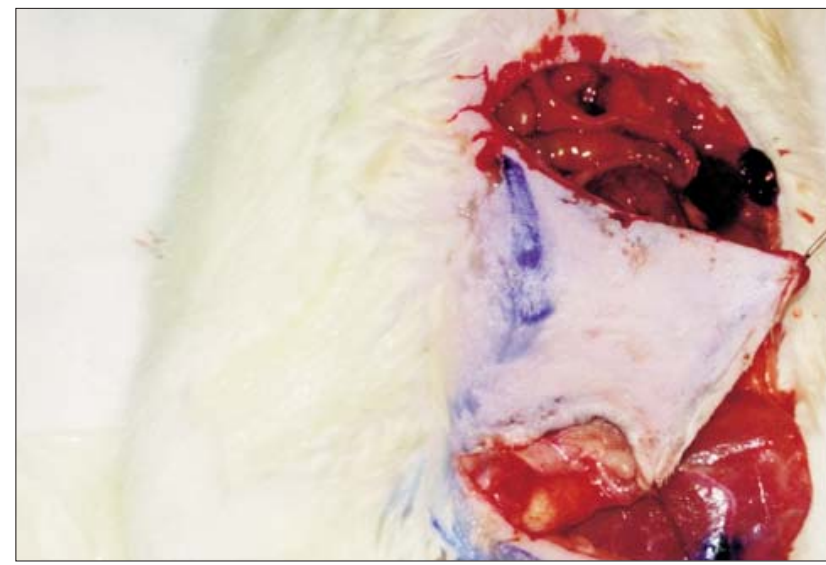

Figure 19 - Marking the flap and incisions of the skin

\section{DISCUSSIONS}

Each of the model presented was tested having in mind its utility for developing immunological studies, which represent a complex research area in the field of composite tissue transplantation.

The suitable model has to be simple, easy to perform, reproducible, ensuring long-term survival of the animals for permitting valid observations in rejection dynamic analysis and possibility of testing different protocols for induction transplant tolerance. We can differentiate between two categories of allografts: allografts including bone in their composition and allografts composed only by skin and soft tissue.

The role of the skin component is to follow-up the rejection, assessing clinical modification and also being a source for biopsies prelevation for histopathology monitoring. The bone component, with its bone

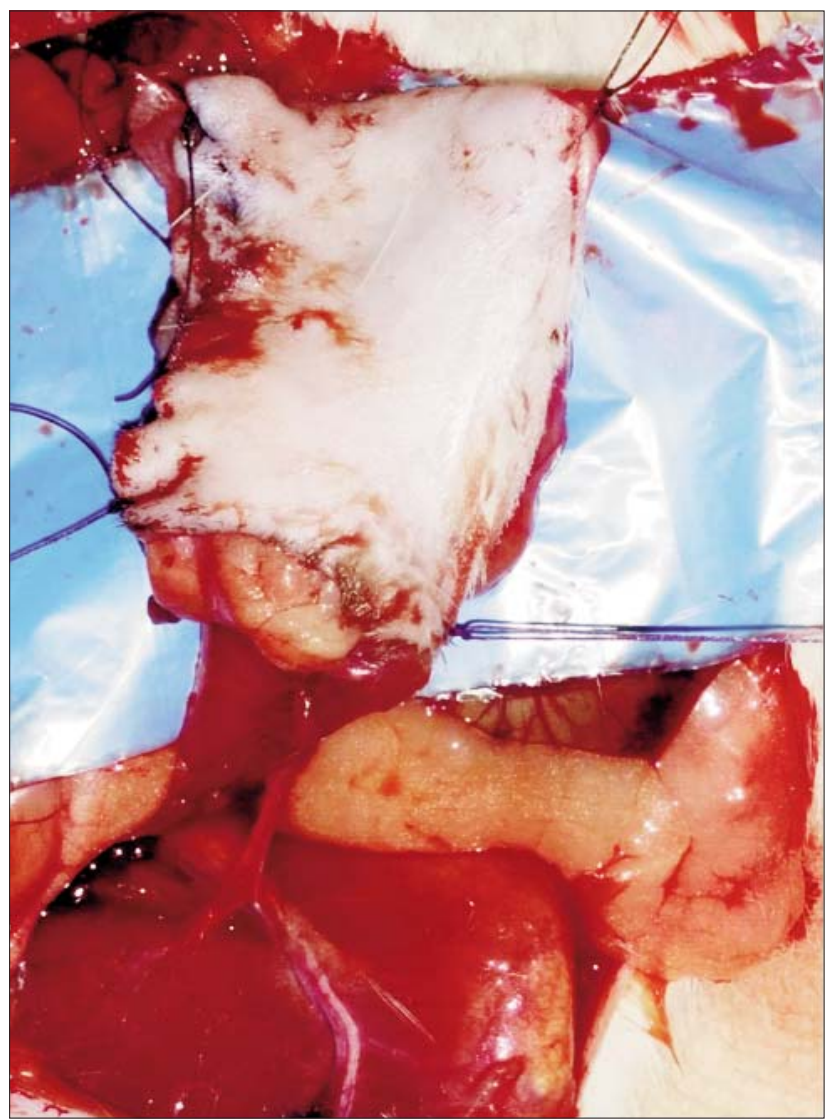

Figure 20 - Raising of the flap

marrow is important as immunomodulatory model trough chimerism induction (15).

Orthotopic hind limb transplantation in rodent model represents the most utilized experimental model in composite tissue transplantation. (18) The model has been used for almost four decades as experimental techniqual model in reconstructive transplantation (19). 


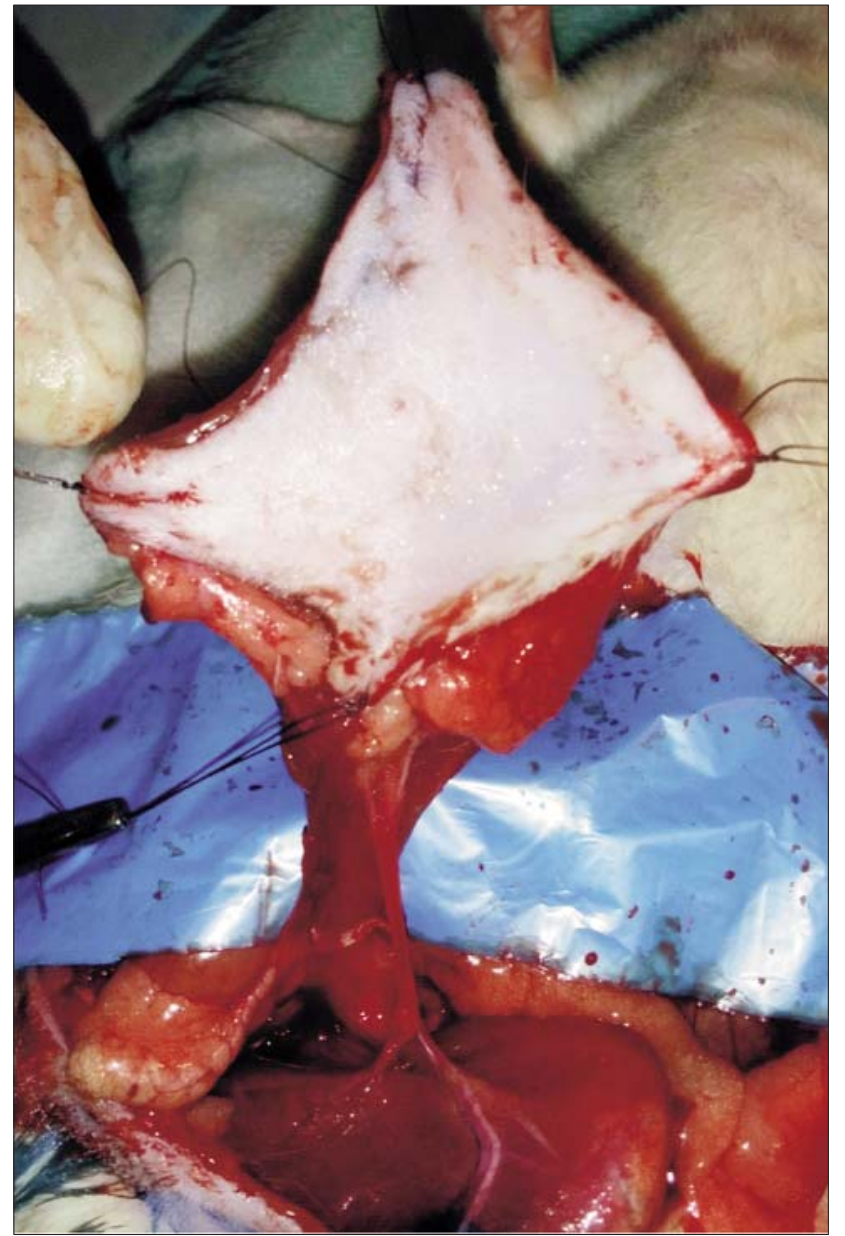

Figure 21 - Dividing the inguinal ligament and raising of the flap basing on iliac vessels

It is a very demanding surgical technique, requiring very good microsurgical skills for performing vascular anastomoses and repair of the nerves (18).

Because it is a very laborious procedure, the orthotopic hind limb allotransplant presents a series of disadvantages:

- Long duration of the intervention;

- It necessitates bone fixation - relatively difficult to be performed and there is a high risk of a femur fracture in case of an inadequate technique. There is a problem in osteosynthesis because of the dimension difference between donor and recipient, with bone incongruence and high risk of consolidation problems at the osteosynthesis site $(10,11)$;

- Intraoperatory/perioperatory complications that may appear: anesthetic toxicity, femur fracture, nerve dilaceration, accidental lesion of the vascular pedicle, hemorrhage, acute arterial thrombosis, venous thrombosis.

- Postoperatory complications: thrombosis, wound dehiscence, infections, autophagy (Lewis rats are
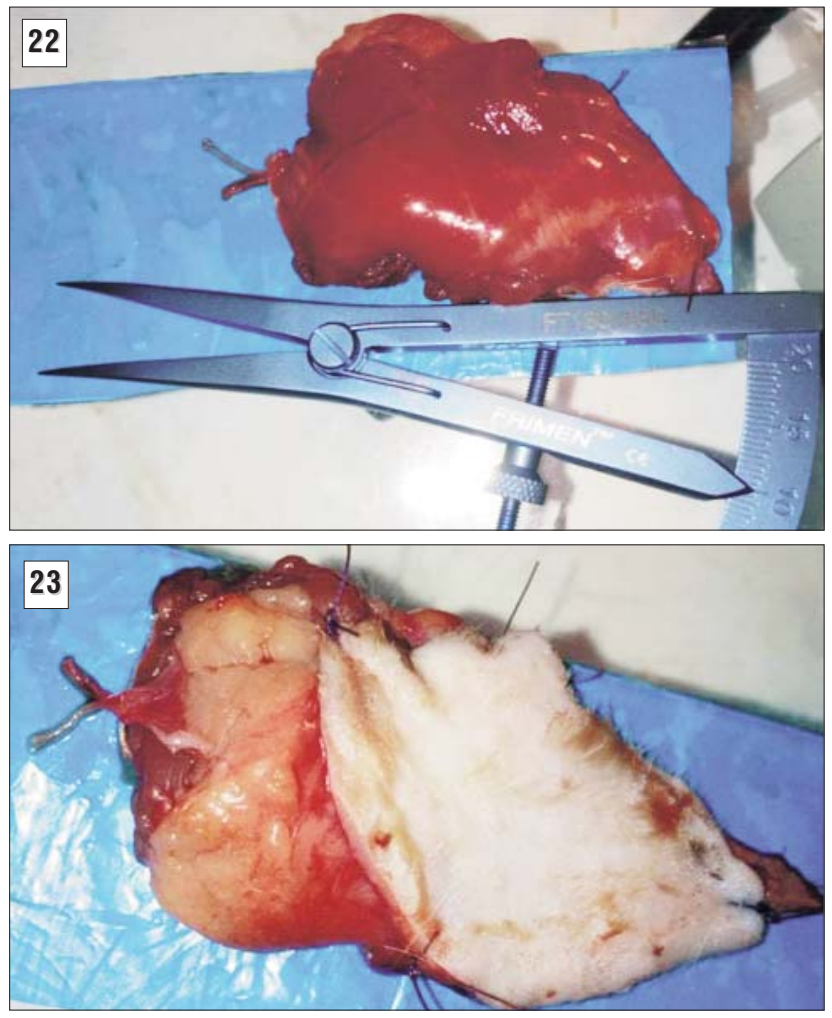

Figures 22, 23 - The aspect of the hemi-abdominal wall flap

more adequate as transplant recipients, being less aggressive) (10).

- Postoperatory (until nervous regeneration and function retrieval), the transplanted limb is not innervated, the animal drags it around, with the appearance of ulcerations, lesions, with the risk of local infections (11).

Comparing with orthotopic hind limb transplant, the postoperatory results of partial hind limb transplant have proven to be favorable in this type of experimental model, without significant complications and being useful for following the dynamics of the post-transplant rejection. The vascular anastomosis implies the femoral vascular pedicles as well, but it is not necessary to repair all the other anatomical elements like in the orthotopic limb transplant.

In the case of the experimental model using the entire hind limb we have noticed the following disadvantages:

- transplant of the entire hind limb results into a much larger dimension of the allograft, that is placed in the inguinal region which determines the rat's discomfort, making its mobility uncomfortable;

- the transplanted limb is easily accessible to the animal which led to its destruction;

- the concomitant dissection of the epigastric flap, 
although it provides a large skin surface, useful for monitoring the rejection, makes the procedure more laborious and elevates the time length of the intervention and the complications risk.

The partial heterotopic transplant has turned out to be a better option for continuing the research on. Certain advantages have been found in the case of using the heterotopic hind limb transplant (compared to the orthotopic one) when immunological studies are involved (10-11):

- it represents a more simple experimental model, from a technical point of view, than the orthotopic model;

- maintaining the bone marrow intact is useful for immunological aspect, knowing the role of microchimerism in immunomodulation and promoting the immune tolerance;

- reducing the morbidity and mortality;

- the possibility of using animals of different sizes.

The osteomyocutaneous flap represents a good alternative for vascularized composite allotransplants based on femoral vessels. The skin island included in the flap's composition is very useful for monitoring the rejection, making this experimental model suitable for immunological studies.

After analyzing the data above one can notice the benefits of using the osteomyocutaneous composite flap over the other procedures(hind limb orthotopic transplantation especially) for composite tissue allotransplantation immunological studies purposes, eliminating difficult technical steps from surgical procedure, with reducing of mean duration of the surgery and ischemia time and avoid the complications related to laborious surgical procedures. Adamson et al., based on their research, considere hind limb heterotopic osteomyocutaneous flap as an ideal experimental model for tolerance induction studies in vascularized composite allotransplantation field (13). Also Zor and Siemionow apreciate a model of groin-thight osteomyocutaneous allotransplant as a good alternative to hind limb transplant models, being a less challenging as surgical technique and associated with low mortality $(15,20)$.

Preserving the intact bone marrow cavity has the utmost importance role regarding the immunomodulation mechanism, trough chimerism induction (21). The variants of hind limb allotransplants presented have bone in their composition, with different quantity of bone marrow included. Studies were conducted for evaluation of the quantity of the bone marrow cells included in different types of allotransplants: the hind limb model contains $48.75 \times 10^{6}$ bone marrow cells and a femur model (described by Tai et al., comprising femoral bone, bone marrow and surrounding soft tissues) containing $50 \times 10^{6}$ bone marrow cells $(15,22)$.

When the immunomodulator role of bone component is not taking in consideration for a specific study, the epigastric flap allograft represents a composite tissue transplantation model very useful for imunological studies due to its skin component( which has an intense immune response) that can well express the rejection mechanism. It also permits taking tissue samples for histopathological studies. The groin flap based on superficial epigastric vessels or femoral vessels is considered technicaly more simple and the transplant procedure is associated with low mortality rates (15). The disadvantage with groin flap is relative small size of skin paddle, aspect which can be overcome when using abdominal wall transplant model.

Abdominal wall transplantation model in rats can provide a useful tool in research of immunological aspects, due to large skin paddle with high antigenic load and stratigraphic particularity of full thickness abdominal wall allograft. The large dimensions of the flap permit good evaluation of skin rejection and different protocols proposed for transplant tolerance induction $(16,23)$.

The dissection is technically difficult and time consuming (requiring high doses of anesthetic) resulting in high morbidity and high mortality risks for the animal. The need of such complex experimental model resides in the high accuracy evaluation of rejection dynamic mechanism due to association of structures contained in the full thickness abdominal wall flap.

\section{CONCLUSIONS}

The hind limb transplantation in rat is an experimental model frequently used in immunological studies regarding the composite tissue transplantation.

The orthotopic hind limb transplantation is a time consuming procedure due to osteosynthesis, it has higher rate of intra and post-operative complications, has the donor-recipient weight/height mismatch and also the highest morbidity. The heterotopic hind limb transplantation has a slightly better outcome, but the best surgical model for immunological studies is transplantation of osteomyocutaneous flaps based of femoral vessels due to significant time saving procedure, low ischemia time, less risk of surgical complications and implicit less morbidity and mortality. For immunological studies that don't require an allograft with bone component in it, the epigastric flap with its skin paddle represents a good alternative making itself useful for 
rejection's specific clinical changes evaluation. The full thickness abdominal wall allotransplantation is a technically difficult experimental model, but with promising benefits in better understanding complex tissue response in allotransplantation.

\section{REFERENCES}

1. Iyer S. Vascularised composite allotransplants: Transplant of upper extremities and face. Indian J Plast Surg. 2015 May-Aug:48(2):111-8.

2. Dubernard JM, Owen E, Herzberg G, Martin X, Guigal V, Dawahra M, et al. The first transplantation of a hand in humans. Early results. Chirurgie. 1999 Sep;124(4):358-65; discussion 365-7. French

3. Huang WC, Lin JY, Wallace CG, Wei FC, Liao SK. Improving the safety of tolerance induction: chimerism and cellular co-treatment strategies applied to vascularized composite allografts. Clin Dev Immunol. 2012;2012:107901.

4. Eun SC. Composite tissue allotransplantation immunology. Arch Plast Surg. 2013 Mar;40(2):141-53.

5. Swearingen B, Ravindra K, Xu H, Wu S, Breidenbach WC, Ildstad ST. Science of composite tissue allotransplantation. Transplantation. 2008 Sep 15;86(5):627-35

6. Khalifian S, Brazio PS, Mohan R, Shaffer C, Brandacher G, Barth RN, et al. Facial transplantation: the first 9 years. Lancet. 2014 Dec 13;384(9960):2153-63.

7. Lee WP, Yaremchuk MJ, Pan YC, Randolph MA, Tan CM, Weiland AJ. Relative antigenicity of components of a vascularized limb allograft. Plast Reconstr Surg. 1991 Mar;87(3):401-11.

8. Ravindra KV, Xu H, Bozulic LD, Song DD, Ildstad ST. The need for inducing tolerance in vascularized composite allotransplantation. Clin Dev Immunol. 2012;2012:438078.

9. Kern B, Sucher R. Small Animal Models for Reconstructive Transplantation. In: Brandacher G. (Ed.). The Science of Reconstructive Transplantation. Springer; 2015

10. Molitor M, Kanatami T, Lanzetta M. Hind-Limb Transplantation in the Rat: Surgical Technique, Anaesthesia and Early Postoperative Management. In: Lanzetta M, Dubernard JM, Petruzzo P (Editors).
Hand Transplantation. Springer; 2007.

11. Ulusal AE, Ulusal BG, Hung LM, Wei FC. Heterotopic hindlimb allotransplantation in rats: an alternative model for immunological research in composite-tissue allotransplantation. Microsurgery. 2005; 25(5):410-4.

12. Nazzal JA, Johnson TS, Gordon CR, Randolph MA, Lee WP. Heterotopic limb allotransplantation model to study skin rejection in the rat. Microsurgery. 2004;24(6):448-53.

13. Adamson LA, Huang WC, Breidenbach WC, Rahhal D, Xu H, Huang $Y$, et al. A modified model of hindlimb osteomyocutaneous flap for the study of tolerance to composite tissue allotransplantation. Microsurgery. 2007;27(7):630-6.

14. Liao TC, Yeh LS, Chu RM, Hou SM. An osteomyocutaneous transplantation model on the rat. Microsurgery. 2001;21(7):329-32.

15. Zor F, Siemionow M. Vascularized Composite Allotransplantation Models. In: Siemionow M (Editor). Plastic and Reconstructive Surgery: Experimental Models and Research Designs. Springer; 2015.

16. Nasir S. Abdominal Wall Transplant Models. In: Siemionow M (Editor). Plastic and Reconstructive Surgery: Experimental Models and Research Designs. Springer; 2015

17. Nasir S, Bozkurt M, Klimczak A, Siemionow M. Large antigenic skin load in total abdominal wall transplants permits chimerism induction. Ann Plast Surg. 2008 Nov;61(5):572-9.

18. Sucher R, Oberhuber R, Margreiter $C$, Rumberg G, Jindal R, Lee WP, et al. Orthotopic hind-limb transplantation in rats. J Vis Exp. $2010 \mathrm{Jul}$ 12;(41). pii: 2022. doi: 10.3791/2022

19. Shapiro RI, Cerra FB. A model for reimplantation and transplantation of a complex organ: the rat hind limb. J Surg Res. 1978 Jun;24(6):501-6.

20. Chang KP, Huang SH, Lin CL, Chang LL, Lin SD, Lai CS. An alternative model of composite tissue allotransplantation: groin-thigh flap. Transpl Int. 2008 Jun;21(6):564-71.

21. Muramatsu K, Doi K, i Tanaka H, Taguchi T. Induction of High-Level Chimerism in Composite Tissue Transplants. In: Lanzetta M, Dubernard JM, Petruzzo P (Editors). Hand Transplantation. Springer; 2007.

22. Tai CY, France MA, Strande LF, Eydelman R, Sheng X, Matthews MS, et al. An extraperitoneal isolated vascularized bone marrow transplant model in the rat. Transplantation. 2003 May 15:75(9):1591-3.

23. Lao WW, Wang YL, Ramirez AE, Cheng HY, Wei FC. A new rat model for orthotopic abdominal wall allotransplantation. Plast Reconstr Surg Glob Open. 2014 May 7;2(4):e136. 